\title{
The Effects of Whole Body Vibration Exercise on Jump Performance and Quadriceps Muscle Activation in Soccer Player
}

\author{
Je-Ho Kim \\ Department of Physical Therapy, Graduate School, Sehan University, Dangjin, Korea
}

Purpose: The purpose of this study was to determine the influence of WBV exercise on CMJ and quadriceps muscle activation according to different frequency of vibration in soccer player and also to find effective frequency for leading appropriate treatment reaction.

Methods: Thirty three subjects were randomly divided into three groups: the three groups are WBV group using $20 \mathrm{~Hz}$ frequency, WBV group using $30 \mathrm{~Hz}$ frequency and squat exercise group according to training method. The exercise program was conducted for six weeks. Subjects were measured on $\mathrm{CMJ}$ and quadriceps muscle activation.

Results: Significant difference in CMJ was observed in the group I, II compared with the group III ( $<<0.05)$. Results of post-hoc, showed a significant difference in CMJ in on group I, II compared with the group III, but no a statistically significant difference between group I and II. Significant difference in quadriceps muscle activation was observed in the group I, II compared with the group III $(p<0.05$, $p<0.01$ ). Results of post-hoc, significant difference in quadriceps muscle activation in on group I, II compared with the group III and significant difference between group I and group II.

Conclusion: This research intervened WBV for soccer players and compared the differences of CMJ and quadriceps muscle activation; as a result of the effective frequency for improving performance, there is a significant difference in $\mathrm{CMJ}$ and quadriceps muscle activation of WBV group with comparison of control group; and it was proved that WBV is effective using $30 \mathrm{~Hz}$ frequency for improving quadriceps muscle activation.

Keywords: Whole body vibration exercise, Jump, Muscle activity, Soccer player

\section{INTRODUCTION}

Jump performance is an ability to move vertically center of mass with the stationary position using muscular strength generated by muscle contraction, and the high jump performance is crucial factor to influence outcome of soccer game. ${ }^{1}$ Vertical jump is a representative test of functional performance, which is evaluate the function of lower extremity is needed interaction of various system, such as recruitment of motor unit, stability of joints, muscular strength, neuromuscular coordination. ${ }^{2}$ Also, it is needed to activate of body and pelvis area and also tight and tibia area muscle in order to represent high level motion and it is shown correlation relative to vertical jump especially for lower extremity muscular strength. Moreover, it is represented high muscle activation when vertical jump for

Received May 26, 2015 Revised Jun 17, 2015

Accepted Jun 18, 2015

Corresponding author Je-Ho Kim

E-mail albam20@naver.com rectus femoris, vastus medialis, vastus lateralis, gluteus muscle and gastrocnemius muscle. ${ }^{3,4}$ When vertical jump, the muscle conducts through stretch-shortening cycle which making concentric contraction with accumulation of elastic energy after eccentric contraction. ${ }^{5}$

The represented training method based on stretch-shortening cycle is plyometric training, which previous researches were reported effective for improvement physical ability, such as muscular strength, vertical jump, quick reaction, and so on, and also for prevention of injuries.$^{6-8}$ However, plyometric training, which is consisted on various jump motion and fast motion of a change of direction, is needed adequate physical conditions, so there is a strong presumption that it is possible to damage muscle and bone system, such as ligaments and tendon, transferring too much load to joints

Copylight (C2015 The Korea Society of Physical Therapy

This is an Open Access article distribute under the terms of the Creative Commons Attribution Non-commercial License (Http:// creativecommons.org/license/by-nc/3.o.) which permits unrestricted non-commercial use, distribution, and reproduction in any medium, provided the original work is properly cited. 
if the condition is not sufficient. ${ }^{9}$

Recently, in order to solve the problem of plyometric training, whole body vibration (WBV), which is a safe exercise method with low intensity, has been introduced; can improve recovery of damaged muscle and bones; and is an effective exercise for performance improvement of patients and athletes with limitation of movement. ${ }^{10-12}$ Mechanism of WBC is stimulate primary ending of muscle spindle with mechanical vibration for muscle, and it is induced the more strong output of alpha motor neuron due to increasing activity of Ia afferent fibers and also contraction of strong muscle with increasing recruitment of motor unit. ${ }^{13}$

It is shown by Armstrong et al. ${ }^{14}$ significant improvement of height of vertical jump after intervention of WBV for 90 subjects and it is resulted by Jordan et al. ${ }^{15}$ significant improvement of muscle activation for quadriceps muscle compared to control group with the result of acute effect of WBV after intervened WBV for one week using $30 \mathrm{~Hz}$ frequency to 24 athletes. Roelants et al. ${ }^{16}$ was reported the significant improvement of muscle activation of rectus femoris, vastus medialis, vastus lateralis and gastrocnemius muscle after intervention of WBV using $35 \mathrm{~Hz}$ frequency when each different squat exercises compared to control group. Also, after intervention of WBV for eight weeks to healthy adults using $20 \mathrm{~Hz}$, it can be shown sufficient improvement of balance ability, so it is reported that WBV, which stimulates mechanoreceptor of joints, can improve balance ability, ${ }^{17}$ and also of pain relief of delayed onset muscle soreness after reinforcement exercise of muscular strength. ${ }^{18}$

Like the above research, WBV is known to be effect, but because it is relatively new method of exercise, it is limited for the research about strengthen, duration, frequency, position, and so on, of vibration for leading appropriate reaction. Therefore, the purpose of research is to investigate the effect influenced to jump performance and quadriceps muscle activation in soccer players with a squatting positon and also to find effective frequency for leading appropriate treatment reaction.

\section{METHODS}

\section{Subject of research}

In this research, the research contents and purpose are sufficiently explained to the subjects and it was conducted for subjects who write agreement of participation. The subjects of this research were
Table 1. General characteristic of subjects

\begin{tabular}{lccc}
\hline & $\begin{array}{c}\text { Group I }(n=11) \\
\text { Mean } \pm \text { SD }\end{array}$ & $\begin{array}{c}\text { Group II }(n=11) \\
\text { Mean } \pm \text { SD }\end{array}$ & $\begin{array}{c}\text { Group III }(n=11) \\
\text { Mean } \pm \text { SD }\end{array}$ \\
\hline Height $(\mathrm{cm})$ & $177.65 \pm 4.46$ & $176.78 \pm 5.18$ & $177.53 \pm 3.23$ \\
Age (year) & $20.54 \pm 1.65$ & $20.49 \pm 1.75$ & $20.42 \pm 1.36$ \\
Weight $(\mathrm{kg})$ & $68.24 \pm 4.13$ & $69.48 \pm 3.46$ & $68.65 \pm 4.55$ \\
\hline
\end{tabular}

Group I, whole body vibration using $20 \mathrm{~Hz}$; Group II, whole body vibration using $30 \mathrm{~Hz}$; Group III, control.

selected 33 soccer players of S university in Jeollanam-Do, and the specific standard of selection is the following (Table 1):

1) A player who do not have medical history in their lower extremity relate to orthopedic surgery

2) A player who do not have any other internal and external disease

3) A player who can do complete weight support

4) A player who do not have motor abnormality and parenthesis

\section{Research procedure}

The purpose of this research is investigated the effect of jump performance and quadriceps muscle activation in soccer player identified the effective vibration frequency leading appropriate remedial reaction with WBV during 6 weeks. The subjects are university soccer players; the pre-test is executed for vertical jump and quadriceps muscle activation; and they were divided into three groups and trained. The three groups, which each group have 11 members, are WBV group using $20 \mathrm{~Hz}$ frequency (Group I), WBV group using 30 $\mathrm{Hz}$ frequency (Group II) and squat exercise group (Group III) according to training method. After 6 weeks, another tests were conducted with similar method to pre-test.

\section{1) WBV}

The participants planted with $8.4 \mathrm{~cm}$ between inner heels of both foot and make their foot narrow with standard distance from rotational axis with a 9 degree relative to valgus angle of great toe. In independent standing position, the squat position was set the previous research of Roelants et al. ${ }^{16}$ as basis and the exercise was intervened in a position of high squat (high squat knee angle $125^{\circ}$, hip angle $140^{\circ}$ ) and low squat (low squat knee and hip angle $90^{\circ}$ ). Using the protocol proposed by van Nes et al. ${ }^{19} \mathrm{WBV}$ was conducted during 6 weeks with every 5 weeks for 20 minutes per a day. For one set, four times of WBV was intervened for 45 seconds, which has two set of high squat and two set of low squat position and also have 1 minute break time between sets. Group I and Group II were applies $20 \mathrm{~Hz}$ 
and $30 \mathrm{~Hz}$, respectively.

\section{2) False WBV}

Group III, which is control group, was applied same situation relative to WBV group except the vibration.

\section{Measurement}

\section{1) Surface electromyogram system}

In order to measure lower extremity muscle activation, MP100 surface electromyogram system (Biopac System Inc., Santa Barbara, CA, USA) was used and the data transferred to digital signals was processed using Acqknowledge 3.91 software in personal computer. For minimizing skin resistance for surface electromyogram signal, the hair of attaching parts were eliminated, rubbed with fine sandpaper for 3-4 times due to getting rid of corneum, and cleaned the skin with sterilization alcohol cotton. The adherence muscle is attached to rectus femoris (RF), vastus lateralis (VL) and vastus medialis (VM) and positive and negative electrodes are adhered with a parallel direction to muscular fibers of muscle belly of each muscle. Sampling rate was set $1,024 \mathrm{~Hz}$, in order to minimize noise, a notch filter and a band pass filter were set $60 \mathrm{~Hz}$ and 30-450 Hz, respectively, and the collected signal was processed with RMS (Figure 1 A).

\section{2) Normalization of muscle activation}

In this research, in order to standardize the muscle activation, \% reference value contraction (\%RVC) method was used, which is a method to standardize based on reference voluntary contraction (RVC) relative to muscle contraction of specific motion. During maintaining 5 seconds a low squatting posture, after it was measured muscle activation of measuring muscle, RVC value was calcu- lated with analyzing signals with 3 seconds excluding first 1 second and last 1 second and also \%RVC value was do with comparing muscle activation value measured during vertical jump. The muscle activation data was used a mean value of acquired results of 3 measurements.

\section{3) Measurement of jump performance}

In order to measure jump performance, G-jump (BTS, Italy) was used. The G-sensor was fixed in 5th spinal puncture of subjects and it was detected the G-sensor value every 2,004 Hz for position, direction, and velocity when jump. These data was analysis using $\mathrm{G}^{-}$ studio software inside personal computer. The protocol was counter movement jump (CMJ) and the data was used mean values of 3 measurements (Figure 1B).

\section{Data analysis}

The measured data was statistically processed using SPSS 19.0; in order to verify normality between three groups, one-way ANOVA was conducted; and it was analyzed using ANCOVA in order to verify the difference of jump performance and quadriceps muscle activation according to each group of method of exercise. Post-hoc comparison was also executed using Bonferroni comparison method. Statistical significance level was $\alpha=0.05$.

\section{RESULTS}

\section{Comparison of jump performance between groups}

As a result of comparison of jump performance, before and after intervene for each group, there was a significant improvement on CMJ jump for group I and group II relative to group III $(\mathrm{p}<0.05)$.
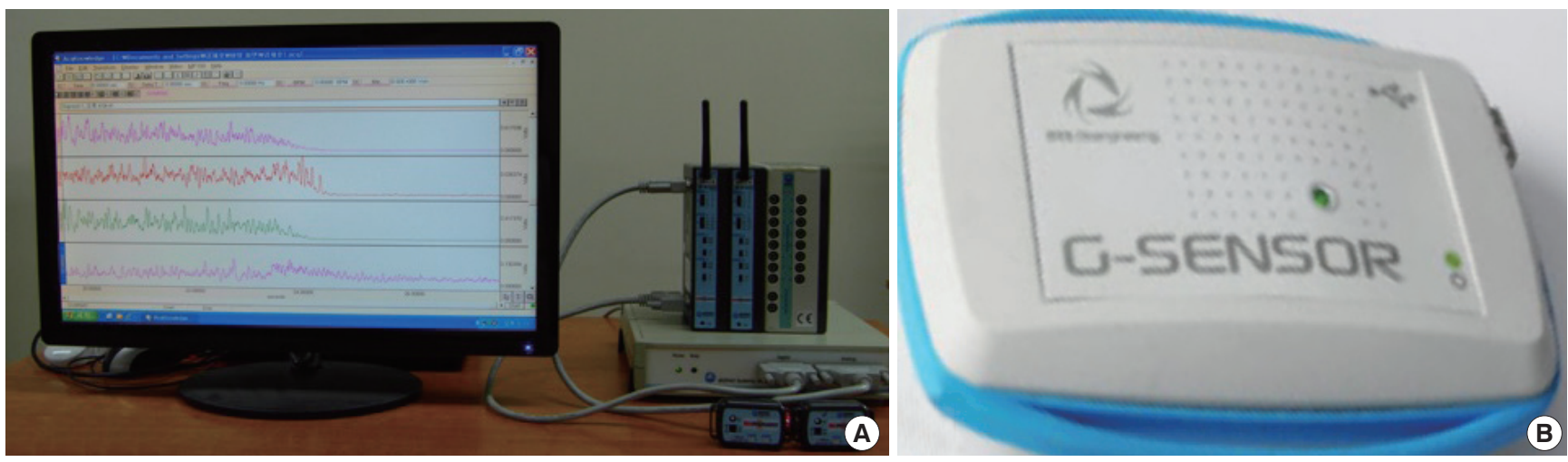

Figure 1. (A) Surface EMG, (B) G-jump. 
Table 2. Comparison of jump performance between groups

(Unit: $\mathrm{cm}$ )

\begin{tabular}{|c|c|c|c|c|c|c|c|c|c|}
\hline & \multicolumn{2}{|c|}{ Group I $(n=11)$} & \multicolumn{2}{|c|}{ Group ॥ $(n=11)$} & \multicolumn{2}{|c|}{ Group III $(n=11)$} & \multirow{2}{*}{$\mathrm{F}$} & \multirow{2}{*}{$p$} & \multirow{2}{*}{ Post-Hoc } \\
\hline & Pre & Post & Pre & Post & Pre & Post & & & \\
\hline \multirow[t]{2}{*}{ CMJ } & $46.72 \pm 4.92$ & $50.95 \pm 3.77$ & $44.43 \pm 3.69$ & $51.37 \pm 4.60$ & $44.28 \pm 4.26$ & $47.13 \pm 3.70$ & 3.36 & $0.048 *$ & $\| I I<1$ \\
\hline & & & & & & & & & $\|I\|<\|$ \\
\hline
\end{tabular}

Group I, whole body vibration using $20 \mathrm{~Hz}$; Group II, whole body vibration using $30 \mathrm{~Hz}$; Group III, control.

$\star p<0.05$.

CMJ, counter movement jump.

Table 3. Comparison of quadriceps muscle activation between groups

(Unit: \%RVC)

\begin{tabular}{|c|c|c|c|c|c|c|c|c|c|}
\hline & \multicolumn{2}{|c|}{ Group I $(n=11)$} & \multicolumn{2}{|c|}{ Group ॥ $(n=11)$} & \multicolumn{2}{|c|}{ Group III $(n=11)$} & \multirow{2}{*}{$\mathrm{F}$} & \multirow{2}{*}{$\mathrm{p}$} & \multirow{2}{*}{ Post-Hoc } \\
\hline & Pre & Post & Pre & Post & Pre & Post & & & \\
\hline \multirow[t]{3}{*}{ RF } & $11.74 \pm 1.92$ & $22.67 \pm 4.01$ & $12.01 \pm 1.69$ & $26.64 \pm 3.84$ & $11.95 \pm 1.26$ & $19.28 \pm 3.70$ & 11.28 & $0.046 *$ & III $<1$ \\
\hline & & & & & & & & & $\|I I<\|$ \\
\hline & & & & & & & & & I $<11$ \\
\hline \multirow[t]{3}{*}{ VM } & $20.78 \pm 2.46$ & $37.56 \pm 4.73$ & $20.48 \pm 2.78$ & $41.31 \pm 5.01$ & $20.53 \pm 2.39$ & $32.28 \pm 4.94$ & 19.49 & $<0.001^{+}$ & III $<$I \\
\hline & & & & & & & & & $\|I I<\|$ \\
\hline & & & & & & & & & $1<11$ \\
\hline \multirow[t]{3}{*}{ VL } & $27.56 \pm 2.13$ & $48.84 \pm 5,87$ & $27.38 \pm .36$ & $52.04 \pm 6.17$ & $27.49 \pm 2.55$ & $42.56 \pm 5.73$ & 23.15 & $<0.001^{+}$ & III $<1$ \\
\hline & & & & & & & & & III $<\|$ \\
\hline & & & & & & & & & $1<11$ \\
\hline
\end{tabular}

Group I, whole body vibration using $20 \mathrm{~Hz}$; Group II, whole body vibration using $30 \mathrm{~Hz}$; Group III, control.

$* p<0.05,{ }^{+} p<0.01$.

$\mathrm{RF}$, rectus femoris; $\mathrm{VM}$, vastus medialis; $\mathrm{VL}$, vastus lateralis.

The result of post-hoc comparison showed statistically significant differences for group I and II relative to group III but no a statistically significant difference between group I and group II (Table 2).

\section{Comparison of quadriceps muscle activation between groups}

As a result of comparison of quadriceps muscle activation, before and after intervene for each group, there was a significant improvement on quadriceps muscle activation for group I and group II relative to group III $(\mathrm{p}<0.05, \mathrm{p}<0.01)$. The result of post-hoc comparison showed statistically significant differences for group I and II relative to group III and also there was a statistically significant difference between group I and group II (Table 3).

\section{DISCUSSION}

WBV is a safe and comfortable exercise with low intensity, which is an exercise method to improve physical function, such as muscular strength, balance, bone density, and so on, and used for various area and diseases recently. This research has purposes of the effect of WBV influenced to jump performance and quadriceps muscle activation of soccer player and also identification of effective frequency to improve performance, and conducted several tests. As results of the research, there was significant improvement on jump performance and quadriceps muscle activation after intervention WBV and also post-hoc comparison result showed that $30 \mathrm{~Hz}$ frequency is effective for improvement of quadriceps muscle activation.

Cochrane et al..$^{20}$ showed that significant difference of jump performance for between a warming up group using bicycles and a hot poultice and a group with WBV for 8 subject with $26 \mathrm{~Hz}$ frequency and also on significant improvement of jump performance of a group using 35-40 Hz WBV relative to false WBV group and resistive exercise group, by Delecluse et al. ${ }^{21}$ conducted for 67 young adult females during 12 weeks.

WBV can give vibration stimulation with amplitude and frequency modulation to muscle; can stimulate muscle spindle in muscle; can improve activation of alpha motor neuron with increasing inputs of Ia afferent fibers; can reinforce circuits of tonic vibration reflex and lead muscle hypertrophy; and can improve jump performance due to increasing reaction of neuromuscular system. ${ }^{22}$

In this research, with comparison between group I and II which are intervened WBV and control group which is intervened false $\mathrm{WBV}$, there is significant difference in jump performance, and the 
similar result of improvement of jump performance to the existing research can support that WBV is necessary for athletes to improve jump performance though there are differences of frequency of vibration stimulation and intervene period.

Turner et al. ${ }^{23}$ showed the significant improvement of jump height at intensity of $40 \mathrm{~Hz}$ compared to $0 \mathrm{~Hz}, 30 \mathrm{~Hz}$, and $35 \mathrm{~Hz}$ after 30 seconds intervention with straight standing position for healthy adult males. In a WBV research of meta-analysis related to chronic effect by Manimmanakorn et al. ${ }^{24}$ there is no significant differences in jump performance according to frequencies over 30 Hz for 15 different papers.

This research also has no significant differences between group I using $20 \mathrm{~Hz}$ and group II using $30 \mathrm{~Hz}$ in a result of post-hoc comparison. Ritzmann et al. ${ }^{25}$ said that it is effective for warm-up due to leading acute effects with short time because neuromuscular system can apply effectively in high frequency, which the higher frequency have, the gather muscle activation is shown. Under $20 \mathrm{~Hz}$ frequency, muscular relaxation is leaded and over $50 \mathrm{~Hz}$ frequency, muscular fatigues can be leaded, so it is necessary to select the frequency according to the purpose of exercise. ${ }^{26}$ Also, it can be thought that there is no significant difference of jump performance according to frequency due to adaptive reaction of neuromuscular system with more than 6 weeks intervention.

In order to improve jump performance, it is needed to be high muscle activation for surrounding muscles, such as pelvis, femur, tibia, and so on, and there is high correlation of muscular strength of rectus femoris, vastus medialis, vastus lateralis, and so on. ${ }^{3,4}$

Jacobs et al. ${ }^{12}$ showed significant differences in extention torque of knee joint of 20 adults, which maintaining a position of knee joint of 170 degree angle during 5 minutes and using $26 \mathrm{~Hz}$ frequency, compared to bicycles training group. Reyes et al. ${ }^{27}$ showed significant improvement in gastrocnemius muscle, biceps femoris muscle, and gluteus muscle activation of 16 baseball players, which intervening WBV, maintaining various positions, and using $30 \mathrm{~Hz}$ frequency, compared to bicycles training group. However, there is no significant difference in muscle activation of vastus lateralis and vastus medialis between with WBV and without WBV for 58 adults using $16 \mathrm{~Hz}$ frequency, bending a knee joint of 60 degree. $^{28}$

In this research, comparing between group I and II with intervening WBV and control group with false WBV intervention, it is shown the significant difference o leg muscle activation and it is concluded that WBV is effective for improving muscle activation, and also Seo and $\mathrm{Kim}^{29}$ said that it is necessary to use frequency more than $20 \mathrm{~Hz}$ for improving muscle activation.

$\mathrm{Kim}^{30}$ showed that it is effective for increasing leg muscle activation for a group with intervening WBV using $30 \mathrm{~Hz}$ vibration frequency as a result of intervention of WBV using $10 \mathrm{~Hz}$ and $30 \mathrm{~Hz}$ vibration frequency for stroke patients. Similar to this, in this research, there are significant differences in increasing leg muscle activation in comparison between group I using $20 \mathrm{~Hz}$ frequency and group II using $30 \mathrm{~Hz}$ frequency as a result of post-hoc comparison.

Thirty $\mathrm{Hz}$ frequency can affect positively in human body due to increasing exercise function, energy metabolism and blood flow rate by attentively increasing of growth hormone, ${ }^{31}$ the action potential can be increased and fatigability can be decreased by increasing excitability of alpha motor neuron and contractible protein sensitivity with increasing inputs of afferent fibers for muscle activation. ${ }^{32}$ Lienhard et al. ${ }^{28}$ suggested that the various change of position considering the physical capability of subjects can be effectively applied muscle activation due to observation of significant increasing for leg muscle activation improvement with a position with bending 60 degree, as a result of WBV intervention using $16 \mathrm{~Hz}$ for position of knee joint with bending 60 degree and comfortability standing position.

This research intervened WBV for soccer players and compared the differences of jump performance and quadriceps muscle activation; as a result of the effective frequency for improving performance, there is a significant difference in jump performance and quadriceps muscle activation of WBV group with comparison of control group; and it was proved that WBV is effective using $30 \mathrm{~Hz}$ frequency for improving quadriceps muscle activation as a result of post-hoc comparison.

The limitation of this research has regional constraints and small number of subjects, so it would be hard to generalize to all soccer players and it is needed to conduct additional research about effect of WBV with various frequency modulation and also with the various position.

\section{ACKNOWLEDGEMENTS}

The Research has been conducted by the Research Grant of Sehan University in 2015. 


\section{REFERENCES}

1. Garstecki MA, Latin RW, Cuppett MM. Comparison of selected physical fitness and performance variables between NCAA Division I and II football players. J Strength Cond Res. 2004;18(2):292-7.

2. Buchanan AS, Docherty CL, Schrader J. Functional performance testing in participants with functional ankle instability and in a healthy control group. J Athl Train. 2008;43(4):342-6.

3. Cheng KB. The relationship between joint strength and standing vertical jump performance. J ApplBiomech. 2008;24(3):224-33.

4. Russell P, Croce RV, Swartz EE, et al. Knee-muscle activation during landing: development and gender comparisons. Med Sci Sports Exerc. 2007;39(1):159-70.

5. Jakobsen MD, Sundstrup E, Randers MB, et al. The effect of strength training, recreational soccer and running exercise on stretch-shortening cycle muscle performance during countermovement jumping. Hum Mov Sci. 2012;31(4):970-86.

6. Carvalho A, Mourao P, Abade E. Effects of strength training combined with specific plyometric exercises on body composition, vertical jump, height and lower limb strength development in elite male handball players: a case study. J Hum Kinet. 2014;41:125-32.

7. Ronnestad BR, Kvamme NH, Sunde A, et al. Short-term effects of strength and plyometric training on sprint and jump performance in professional soccer players J Strength Cond Res. 2008;22(3):773-80.

8. Twist C, Eston R. The effects of exercise-induced muscle damage on maximal intensity intermittent exercise performance. Eur J Appl Physiol. 2005;94(5-6):652-8.

9. Jensen RL, Ebben WP. Quantifying plyometric intensity via rate of force development, knee joint, and ground reaction forces. J Strength Cond Res. 2007;21(3):763-7.

10. Mikhael M, Orr R, Fiatarone Singh MA. The effect of whole body vibration in older adults: A systematic review of the literature. Maturitas. 2010;66(2):150-7.

11. Ebersbach G, Edler D, Kaufhold O, et al. Whole body vibration versus conventional physiotherapy to improve balance and gait in parkinson's disease. Arch Phys Med Rehabil. 2008;89(3):399-403.

12. Jacobs PL, Burns P. Acute enhancement of lower-extremity dynamic strength and flexibility with whole-body vibration. J Strength Cond Res. 2009;23(1):51-7.

13. Shinohara M. Effects of prolonged vibration on motor unit activity and motor performance. Med Sci Sports Exerc. 2005;37(12):2120-5.

14. Armstrong WJ, Grinnell DC, Warren GS. The acute effect of wholebody vibration on the vertical jump height. J Strength Cond Res. 2010; 24(10):2835-9.

15. Jordan M, Norris S, Smith D, et al. Acute effects of whole-body vibration on peak isometric torque, muscle twitch torque and voluntary muscle activation of the knee extension. Scand J Med Sci Sports. 2010;20(3): 535-40.

16. Roelants M, Verschueren SM, Delecluse C, et al. Whole body vibration induced increase in leg muscle activity during different squat exercises. J Strength Cond Res. 2006;20(1):124-9.
17. Hiroshige K, Mahbub MH, Harada N. Effects of whole-body vibration on postural balance and proprioception in healthy young and elderly subjects: a randomized cross-over study. J Sports Med Phys Fitness. 2014;54(2):216-24.

18. Kim ES, Kim MW, Cho YM. The effect of vibration training pre-eccentric exercise on delayed onset muscle soreness of triceps surae. J Kor Aca Ind Coop Soc. 2011;12(12):5789-96.

19. van Nes IJ, Latour H, Schils F, et al. Long term effects of 6week whole body vibration on balance recovery and activities of daily living in the post acute phase of stroke: a randomized, controlled trial. Stroke. 2006; 37(9):2331-5.

20. Cochrane DJ, Stannard SR, Sarqeant AJ, et al. The rate of muscle temperature increase during acute whole body vibration exercise. Eur J Appl Physiol. 2008;103(4):441-8.

21. Delecluse C, Roelants M, Verschuren S. Strength increase after whole body vibration compared with resistance training. Med Sci Sports Exerc. 2003;35(6):1033-41.

22. Mischi M, Cardinale M. The effects of a $28 \mathrm{~Hz}$ vibration on arm muscle activity during isometric exercise. Med Sci Sports Exerc. 2009;41(3):645-53.

23. Turner AP, Sanderson M, Attwood LA. The acute of different frequencies of whole body vibration on countermovement jump performance. J Strength Cond Res. 2011;25(6):1592-7.

24. Manimmanakorn N, Hamlin MJ, Ross JJ, et al. Long term effect of whole body vibration training on jump height: meta analysis. J Strength Cond Res. 2014;28(6):1739-50.

25. Ritzmann R, Gollhofer A, Kramer A. The influence of vibration type, frequency, body position and additional load on the neuromuscular activity during whole body vibration. Eur J Appl Physiol. 2013;113(1):1-11.

26. Prisby RD, Lafage-Proust MH, Malaval L, et al. Effects of whole body vibration on the skeleton and other organ system in man and animal model: what we know and what we need to know. Ageing Res Rew. 2008;7(4):319-29.

27. Reyes GF, Dickin DC, Crusat NJ, et al. Whole body vibration effects on the muscle activity of upper and lower body muscles during the baseball swing in recreational baseball hitters. Sports Biomech. 2011;10(4):280-93.

28. Lienhard K, Vienneau J, Friesenbichler B et al. The effect of whole body vibration on muscle activity in active and inactive subjects. Int J Sports Med. 2015;11.

29. Seo HJ, Kim JH. Analysis of muslcle activation related to postural stability according to different frequency of whole body vibration during quiet standing. J Kor Phys Ther. 2013;25(5):316-21.

30. Kim JH. The effects of whole body vibration exercise on balance and lower extremity muscle activity in stroke patients. J Kor Phys Ther. 2013; 25(5):316-21.

31. Cardinale M, Soiza RL, Leiper JB, et al. Hormonal responses to a single session of whole body vibration exercise in older individuals. Br J Sports Med. 2010;44(4):284-8.

32. Hodgson M, Docherty D, Robbins D. Post-activation potentiation: underlying physiology and implications for motor performance. Sports Med. 2005;35(7):585-95. 